\title{
A FAMILY OF GROUPS WITH NICE WORD PROBLEMS
}

\author{
Dedicated to the memory of Hanna Neumann
}

VERENA HUBER DYSON

(Received 27 June 1972)

Communicated by M. F. Newman

This paper is an outgrowth of my old battle with the open sentence problem for the theory of finite groups. The unsolvability of the word problem for groups (cf. [1] and [4]) entails the undecidability of the open sentence problem for the elementary theory of groups and thus strengthens the original undecidability result for this theory (cf. [7]). The fact that the elementary theory of finite groups is also undecidable (cf. [2] and [6]) therefore justifies my interest in the open sentence problem for that theory. This paper contains a construction of groups that might lead to a negative solution.

With every set $S$ of integers is associated a finitely generated group $L(S)$ with the following properties:

(i) $L(S)$ has a solvable word problem if and only if $S$ is recursive, and

(ii) the largest residually finite epimoprhic image of $L(S)$ is isomorphic to $L\left(S^{*}\right)$, where $S^{*}$ denotes the closure of $S$ in the ideal topology on the ring of integers.

For an appropriate choice of the set $C, L\left(C^{*}\right)$ is re presentable and residually finite but has an unsolvable word problem. A suitable choice of the set $D$ yields a group $L(D)$ that has a solvable word problem while the group $L\left(D^{*}\right)$ has an unsolvable word problem, although the set of non-trivial elements of $L\left(D^{*}\right)$ is recursively enumerable. A modification of $D$ leads to an re presentable group $L(E)$, for which the set of trivial words is recursively inseparable from the set of non-trivial ones of $L\left(E^{*}\right)$. A so-called finitary embedding of $L(D)$ into a finitely presented group would establish the undecidability of the open sentence problem for the theory of finite groups. A finitary embedding of $L(E)$ into a finitely presented group would

This research has been supported by the National Research Council of Canada Grant while the author was at the University of Calgary. 
lead to the recursive inseparability of the set of open sentences true in all groups from the set of open sentences whose negations are satisfiable in finite groups. $L(C)$ shows that not every decidable group is so embeddable. However, $L(D)$ and $L(E)$ do satisfy an obviously necessary condition for finitary embeddability and the question arises whether this condition might also be sufficient.

The groups $L(S)$ are amalgamated products of two copies of the restricted wreath product of the group of order two by the infinite cyclic group. They are transparent enough to yield examples and counterexamples in many contexts.

\section{Notation, definitions and generalities}

If $X$ is a set we denote by $F(X)$ the free group generated by $X$. A triple, $\langle X, W, \pi\rangle$, is asid to present the group $G$ if $W \subset F(X)$ and $\pi: X \rightarrow G$ is a set map whose natural extension $\bar{\pi}: F(X) \rightarrow G$ is an epimorphism, with kernel $\langle\langle W\rangle\rangle$, where $\langle\langle W\rangle\rangle$ is the normal closure of $W$ in $F(X)$. If $\pi$ is the inclusion $X \rightarrow F(X)$ we simply write $\langle X ; W\rangle$ and identify this canonical presentation with the group $F(X) /\langle\langle W\rangle\rangle$. If $X$ is countable then any enumeration of $X$ gives rise to various intuitively effective bijections $\gamma$ of $F(X)$ onto the set $\omega$ of natural numbers. We shall from now on consider $X$ to be countable and fix such a bijection $\gamma$. If $U \subset F(X)$ and $\gamma(U)$ is recursive or recursively enumeable, or is the complement of a recursively enumerable set, then accordingly $U$ is called recursive, re or co-re. A presentation $\langle X, W, \pi\rangle$ is said to be recursive, re or co-re if its kernel $\langle\langle W\rangle\rangle$ has the corresponding property. If $\langle X, W, \pi\rangle$ presents the group $G, U \subset G$ and the preimage of $U$ under $\pi$ is recursive, re or co-re, then $U$ is called $\pi$-recursive, $\pi$-re or $\pi$-co-re respectively. It is clear now what we mean by a recursively, re ore co-representable group. Observe, however, that a recursively presented group in our sense has a solvable word problem, while what we call an re presentable group would often be called a recursively presentable one. The reason for this lies in the fact that, for an re set $W$, one can always find a recursive set $W^{\prime}$ such that $\langle\langle W\rangle\rangle=\left\langle\left\langle W^{\prime}\right\rangle\right\rangle$. We have chosen our terminology because it is really the normal closure $\langle\langle W\rangle\rangle$ that matters. If $G$ is finitely generated then all the above properties are independent of the particular choice of a presentation $\langle X, W, \pi\rangle$ for $G$, provided $X$ is finite. We then simply talk about re, co-re or recursive groups and subgroups. If both $X$ and $W$ are finite then the presentation $\langle X, W, \pi\rangle$ is called finite. We shall only be concerned with finitely generated groups.

Let $\mathscr{K}$ be a class of groups closed under isomorphisms. We denote by $\mathscr{R} \mathscr{K}$ the class of all residually $\mathscr{K}$ groups, i.e., the subcartesian closure of $\mathscr{K}$. For any group $G$ we set

$$
\begin{aligned}
M_{\mathscr{X}}(G) & =\cap\{N \triangleleft G \mid G / N \in \mathscr{K}\} \\
K G & =G / M_{\mathscr{X}}(G) .
\end{aligned}
$$


Then $K G$ is the largest epimorphic image of $G$ that belongs to $\mathscr{R} \mathscr{K}$, and $G \in \mathscr{R} \mathscr{K}$ if and only if $G=K G$. If $\mathscr{K}$ contains with a group also all its subgroups, then $\mathscr{R} \mathscr{K}$ is a reflective subcategory of the category $\mathscr{G}$ of all groups with reflector $\boldsymbol{K}$ and unit can: $G \rightarrow G / M_{\mathscr{X}}(G)$. Though the functor $\boldsymbol{K}$ obviously preserves epimorphisms it does not in general preserve monomorphisms, since $U<G$ only implies $M_{\mathscr{K}}(U)<M_{\mathscr{K}}(G) \cap U$. We call a monomorphism $\phi: G \rightarrow H \mathscr{K}$-proper if $\boldsymbol{K} \phi: \boldsymbol{K} G \rightarrow \boldsymbol{K} H$ is monic, i.e., if

$$
\phi\left(M_{\mathscr{K}}(G)\right)=M_{\mathscr{K}}(H) \cap \phi(G) .
$$

Moreover, if $W \subset F(X)$, we set

$$
\langle\langle W\rangle\rangle_{\mathscr{X}}=\cap\{N \triangleleft F(X) \mid W \subset N, F(X) / N \in \mathscr{K}\} .
$$

$\langle X, W, \pi\rangle$ is said to $\mathscr{K}$-present $H \in \mathscr{R} \mathscr{K}$ (or to present $H$ as an $\mathscr{R} \mathscr{K}$-group) if the natural extension of $\pi: X \rightarrow H$ has kernel $\langle\langle W\rangle\rangle_{\mathscr{X}}$, i.e., if $\pi$ induces an isomorphism $K\langle X, W\rangle \cong H$. The concepts of recursiveness etc. introduced above carry over to $\mathscr{K}$-presentations in an obvious way.

We now define the open sentence problem for the class $\mathscr{K}$. The universal closure of any Boolean combination of equations is called an open sentence. The Boolean operations used here are those of conjunction, disjunction, negation and, for convenience, also implication. Of particular interest to us are among them the so-called conditional equations, i.e., open sentences of the form

$$
\Sigma \equiv\left(\forall x_{1}\right) \cdots\left(\forall x_{n}\right)\left(w_{1}=1 \& \cdots \& w_{k}=1 \Rightarrow w=1\right) .
$$

where the $w_{i}^{\prime}$ s and $w$ are words on the variables $x_{1}, \cdots, x_{n}$ and their formal inverses $x_{i}^{-1}$. The open sentence problem for the class $\mathscr{K}$ is said to be solvable (decidable) if and only if there exists a uniform algorithm for deciding whether or not an open sentence holds for all groups $G \in \mathscr{K}$; in other words, if and only if the set of open sentences valid for $\mathscr{K}$ is recursive. We observe that the existence of a proof procedure means that the set is re, and that of a disproof procedure means that it is co-re. The conditional equation problem is defined similarly. It is clear that the unsolvability of the conditional equation problem entails the unsolvability of the open sentence problem. If the class is closed under direct products, then the two problems coincide. We collect a few easily verified facts:

(1) If $G \in \mathscr{R} \mathscr{K}$, then every conditional equation that is valid in $\mathscr{K}$ holds in $G$.

(2) If $\mathscr{K}$ is closed under finite direct products and $G \in \mathscr{R} \mathscr{K}$ then every open sentence that is valid in $\mathscr{K}$ holds in $G$.

Now let $X=\left\{x_{1}, \cdots, x_{n}\right\}, W=\left\{w_{1}, \cdots, w_{k}\right\}$ and let Cons ${ }_{\mathscr{X}} W$ stand for the set of all words $w \in F(X)$ for which the sentence $\Sigma$ above is valid in $\mathscr{K}$. If $w \notin$ Cons $_{\mathscr{X}} W$ then there exists a homomophism of $F(X)$ into a $\mathscr{K}$-group, whose kernel contains $W$ but not $w$. Thus, if $\mathscr{K}$ contains with a group also all its subgroups, $w \notin\langle\langle W\rangle\rangle_{\boldsymbol{x}}$. From this together with (1) follows: 
(3) If $\mathscr{K}$ is closed under subgroups then Cons $_{\mathscr{X}} W=\langle\langle W\rangle\rangle_{\mathscr{K}}$.

Observing that $K G \cong F(X) /\langle\langle W\rangle\rangle_{\mathscr{X}}$ if $G$ has a presentation $\langle X, W, \pi\rangle$, we obtain from this

Proposition 1. Let the class $\mathscr{H}$ of groups be closed under subgroups.

(i) A finitely presented group $G$ belongs to $\mathscr{R} \mathscr{K}$ if and only if all conditional equations that are valid for $\mathscr{K}$ hold in $G$.

(ii) If there exists a finitely presented group $G$ for which $K G$ has an unsolvable word problem, then the conditional equation problem and hence also the open sentence problem for $\mathscr{K}$ is undecidable.

We shall only be concerned with the class $\mathscr{F}$ of finite groups and use the following terminology: $M_{f}(G)=M_{\mathscr{F}}(G)$ is the finitary kernel of $G$ and $G_{f}=F G$ is its finitary image, $\langle\langle W\rangle\rangle_{f}=\langle\langle W\rangle\rangle_{F}$ is the finitary kernel of the presentation $\langle X ; W\rangle$, and an $\mathscr{F}$-proper monomorphism is a finitary embedding.

A few trivial considerations and the observation that a list of all finite multiplication tables yields an effective enumeration of all finite groups which uniformly displays decision procedures for their word problems lead to the following (cf. [5])

Proposition 2. (i) Every finitely generated subgroup of a recursive group is recursive.

(ii) A group that is embeddable in a finitely presented group is re.

(iii) If a group $G$ is finitarily embeddable in a finitely presentable group then $G_{f}$ is co-re.

(iv) If a residually finite group $H$ is embeddable in a residually finite group that is finitely presentable as a residually finite group, then $H_{f}$ is co-re.

COROLLARY. Every finitely presentable residually finite group has a solvable word problem. In particular, every finitely generated nilpotent group has a solvable word problem and so does every finitely generated metabelian group.

Note that finitely generated nilpotent or metabelian groups satisfy the maximal condition on normal subgroups and are thus finitely presentable (cf. [3]).

We recall that Higman's remarkable result (cf. [4]) proves the unsolvability of the word problem for groups by establishing the converse of (ii). The construction of an re presentation $\langle X ; W\rangle, X$ finite, for which $\langle\langle W\rangle\rangle$ is recursively inseparable from the complement $F(X) \mid\langle\langle W\rangle\rangle_{f}$, which also is re, leads to the question, whether the converses of (iii) and (iv) might also be true, i.e.,

PRoblem 1. Is every re group $G$ with co-re finitary image $G_{f}$ finitarily embeddable in a finitely presentable group?

PROBLEM 2. Is every residually finite co-re group embeddable in a residually finite group that is finitely presentable as a residually finite group? 
A positive answer to either problem would establish the undecidability of the open sentence problem for the theory of finite groups. A positive answer to Problem 1 would entail the recursive inseparability of the set of conditional equations valid for all groups from the set of those that fail in some finite groups.

\section{The groups $L(S)$}

We use the customary notation for presentations and denote by $\langle U\rangle_{G}$ and $\langle\langle U\rangle\rangle_{G}$ the subgroup and the normal subgroup of $G$ generated by the subset $U$ of $G$, respectively. Whenever there is no danger of ambiguity we omit the subscripts. By $\gamma_{m}(G)$ we denote the $m$-th term of the lower central series of $G$, i.e., $\gamma_{0}(G)=$ $G, \gamma_{n+1}(G)=\left[\gamma_{n}(G), G\right]$. Moreover we set:

$$
[x, y(0)]=x, \quad[x, y(n+1)]=[[x, y(n)], y],
$$

$A=\langle a\rangle$, the infinite cyclic group generated by $a$,

$A_{n}=\left\langle a ; a^{n}\right\rangle$, the cyclic group of order $n$ generated by $a$,

$B_{0}=\left\langle b ; b^{2}\right\rangle$, the group of order 2 generated by $b$,

$L=\left\langle a, b ;\left\{b^{2},\left[a^{i}, b\right]^{2} \mid i \in Z\right\}\right\rangle \simeq B_{0} 2 A$,

$L_{n}=\left\langle a, b ;\left\{b^{2}, a^{n},\left[a^{i}, b\right]^{2} \mid 0<i<n\right\} \simeq L /\left\langle\left\langle a^{n}\right\rangle\right\rangle \simeq B_{0} \prec A_{n}\right.$,

$b_{i}=a^{-i} b a^{i}$,

$B=\langle\langle b\rangle\rangle_{L} \triangleleft L$, the base group of $L$, generated by the $b_{i}$ 's,

$B_{n}=\langle\langle b\rangle\rangle_{L_{n}} \triangleleft L_{n}$, the base group of $L_{n}$.

Note that

$$
\begin{aligned}
L & =\left\langle B, a ;\left\{b_{i+1} b_{i}^{a} \mid i \in Z\right\}\right\rangle, \\
L_{n} & =\left\langle B_{n}, A_{n} ;\left\{b_{i+1} b_{i}^{a} \mid 0 \leqq i<n\right\}\right\rangle
\end{aligned}
$$

Every element $l$ of $L$ or $L_{n}$ has a unique normal form with $I=Z$ or $\{0, \cdots, n-1\}$, respectively:

(1) $l=a^{k} \prod_{i \in I} b_{i}^{v_{i}}, k \in I, v_{i}=0$ or 1,0 for all but finitely many $i$,

$$
l^{\prime}=a^{k+k^{\prime}} \prod_{i \in I} b_{i}^{v_{i-k^{\prime}+v_{i}^{\prime}}}
$$

where the exponents of the $b_{i}$ are to be taken modulo 2, and, in the case of $L_{n}$, the exponent of $a$ is to be taken modulo $n$. It follows that $L$ and $L_{n}$ have solvable word problems.

Let $\alpha, \alpha^{\prime} \in A$ (or $A_{n}$ ), $\beta, \beta^{\prime} \in B$ (or $B_{n}$ ), then we have

$$
\begin{gathered}
{\left[l, l^{\prime}\right]=\left[\alpha \beta, \alpha^{\prime} \beta^{\prime}\right]=\left[\beta, \alpha^{\prime}\right]\left[\beta^{\prime}, \alpha\right]=\left[l^{\prime}, l\right] \in B \quad\left(\text { or } B_{n}\right),} \\
{\left[\beta \beta^{\prime}, \alpha\right]=[\beta, \alpha]\left[\beta^{\prime}, \alpha\right] .}
\end{gathered}
$$


Moreover, for all $i, k \in Z$,

(5) $\left[b_{b}, a^{k}\right]=b_{i} b_{i+k}, \quad\left[b_{i}, a^{k+1}\right]=\left[b_{i}, a^{k}\right]\left[b_{i+k}, a\right]$ and $\left[b_{i}, a^{-k}\right]=\left[b_{i-k}, a^{k}\right]$,

$$
\left[\beta, \alpha \alpha^{\prime}\right]=\left[\beta, \alpha, \alpha^{\prime}\right][\beta, \alpha]\left[\beta, \alpha^{\prime}\right] \text {, }
$$

in particular

$$
\left[\beta, \alpha^{2}\right]=[\beta, \alpha(2)]
$$

and thus by induction

$$
\left[\beta, \alpha^{2^{r}}\right]=\left[\beta, \alpha\left(2^{r}\right)\right] \in \gamma_{2^{r}}(L) .
$$

It follows from (3), (4) and (5) that

$$
\gamma_{m}(L)=\langle\langle[b, a(m)]\rangle\rangle=\left\langle\left[b_{i}, a(m)\right] \mid i \in Z\right\rangle
$$

freely generated as an elementray abelian 2-group.

$$
\left|B / \gamma_{m}(L)\right|=2^{m} \text {. }
$$

From (8) and (9)

$$
\begin{gathered}
\gamma_{2^{r}}\left(L_{n}\right)=\gamma_{2^{r+1}}\left(L_{n}\right) \quad \text { for } n=2^{r} q, \quad q \text { odd. } \\
\left\langle\left\langle a^{n}\right\rangle\right\rangle \cap B=\left\langle\left\langle b b_{n}\right\rangle\right\rangle=\left\langle b_{i} b_{i+n} \mid i \in Z\right\rangle=\underset{i \in Z}{X}\left\langle b_{i} b_{i+n}\right\rangle .
\end{gathered}
$$

We collect some conclusions from all this:

LeMmA 1. (i) If $n=2^{k}$ then $L_{n}$ is nilpotent of class $n$.

(ii) If $n=2^{k} q, 1 \neq q$ odd, then the intersection of the lower central series of $L_{n}$ coincides with its $2^{k}$-th term and is non-trivial.

(iii) $L$ is metabelian, and so is $L_{n}$.

(iv) $L$ is residually an $\left\{L_{n} \mid n \in U\right\}$-group for every infinite set $U$ of positive integers. In particular $L$ is residually finite non-nilpotent as well as residually a finite 2-group.

We now proceed to construct a group $L(S)$ for every set $S$ of integers. Let $L^{\prime}$, generated by $\left\{a^{\prime}, b^{\prime}\right\}$, be an isomorphic copy of $L$ with the isomophism $\sigma: L \rightarrow L^{\prime}$ induced by $a \leftrightarrow a^{\prime}, b \leftrightarrow b^{\prime}$. Given a set $S \subseteq Z$ we denote by $\sigma(S)$ the restriction of $\sigma$ to the subgroup $J(S)=\left\langle b_{i} \mid i \in S\right\rangle$. We define $L(S)$ as the free product of $L$ and $L^{\prime}$ with the subgroups $J(S)$ and $J^{\prime}(S)$ amalgamated under $\sigma(S)$, i.e.,

$$
L(S)=\underset{\sigma(S)}{L * L^{\prime}}=\left\langle L, L^{\prime} ;\left\{b_{i} b_{i}^{\prime} \mid i \in S\right\}\right\rangle .
$$

Similarly we define $L_{n}(S)$ for $S \subseteq\{0, \cdots, n-1\}$. As an amalgamated product of finite groups $L_{n}(S)$ is residually finite. Note that if $S \subset T$ then $L(T)$ is a proper 
epimorphic image of $L(S)$. We shall establish a criterion for the residual finiteness of $L(S)$. For this purpose we need

LEMMA 2. Given groups $G$ and $H$ with subgroups $U<G$ and $V<H$ and an isomorphism $\sigma: U \rightarrow V$. Assume that $\mathscr{N}$ and $\mathscr{M}$ are families of normal subgroups of $G$ and $H$, respectively, satisfying the following conditions:

(i) $G / N$ and $H / M$ are finite for all $N \in \mathscr{N}, M \in \mathscr{M}$

(ii) to each $N \in \mathscr{N}$ there exists $M \in \mathscr{M}$ such that $\sigma$ restricts to an isomorphism $U \cap N \rightarrow V \cap M$, and vice versa,

(ii) $\cap \mathscr{N}=1$ and $\cap \mathscr{M}=1$,

(iv) $\cap\{U N \mid N \in \mathscr{N}\}=U$ and $\cap\{V M \mid M \in \mathscr{M}\}=V$.

Then the amalgamated product $G_{\sigma}^{*} H$ is residually finite.

Proof. If $1 \neq w=u g_{1} h_{1} \cdots g_{n} h_{n}$ in canonical form, then we can choose $N_{0}, \cdots, N_{n}$ and $M_{1}, \cdots, M_{n}$ such that $u \notin N_{0} \in \mathcal{N}$ or $N_{0}=G$ (if $u=1$ ), $g_{1} \notin U N_{1}$ with $N_{1} \in \mathscr{N}$ or $N_{1}=G$ (if $g_{1}=1$ ), $g, \notin U N_{j}$ with $N_{j} \in \mathscr{N}$ for $j=2, \cdots, n$, $h_{i} \notin V M_{i}$ with $M_{i} \in \mathscr{M}$ for $i=1, \cdots, n-1$, and $h_{n} \notin V M_{n}$ with $M_{n} \in \mathscr{M}$ or $M_{n}=H$ (if $h_{n}=1$ ). Since $w \neq 1$ at least one of $N_{0}, \cdots N_{n}, M_{1}, \cdots, M_{n}$ will belong to $\mathscr{N}$ or $\mathscr{M}$. Now pick $M_{j}^{\prime}, j=0, \cdots, n$ and $N_{i}^{\prime}, i=1, \cdots, n$, so that $\sigma$ restricts to isomorphisms $N_{j} \cap U \rightarrow M_{j}^{\prime} \cap V, N_{i}^{\prime} \cap U \rightarrow M_{i} \cap V$, for $N_{j} \neq G, M \neq H$ and set $G^{\prime}=H, H^{\prime}=G$. Then the normal subgroups

and

$$
K=N_{0} \cap \cdots \cap N_{n} \cap M_{1}^{\prime} \cap \cdots \cap M_{n}^{\prime}
$$

$$
J=N_{0}^{\prime} \cap \cdots \cap N_{n}^{\prime} \cap M_{1} \cap \cdots \cap M_{n}
$$

are of finite index in $G$ and $H$, respectively, and $\sigma$ induces an isomorphism $\sigma^{\prime}: U K / K \rightarrow V J / J$. But $z x_{1} y_{1} \cdots x_{r} y_{r} \mapsto \tilde{z} \tilde{x}_{1} \bar{y}_{1} \cdots \tilde{x}_{r} \bar{y}_{r}$, where $x \mapsto \tilde{x}$ and $y \mapsto \bar{y}$ denote the canonical maps $G \rightarrow G / K$ and $H \rightarrow H / J$, defines an epimorphism $G \underset{\sigma}{*} H \rightarrow(G / K) \underset{\sigma}{*}(H / J)$. Since it preserves the normal form length of $w$ and its image is an amalgamated product of finite groups, and so is residually finite, the lemma is proved.

Now a set $S \subset Z$ is closed under the ideal topology of $Z$ if and only if its complement is open, i.e., if $Z \backslash S$ is the union of a family of arithmetic progressions,

$$
Z \backslash S=\bigcup\{h+k Z \mid(h, k) \in R\}
$$

for some set $R \subseteq Z \times(Z \backslash\{0\})$. We denote the closure of $S$ by $S^{*}$. The complement $Z \backslash S^{*}$ of $S^{*}$ is then the union of all residue classes contained in the complement of $S$. We observe that $S^{*}$ is the intersection of all preimages of $S$ under proper epimorphisms of $Z$, or, using self-explanatory notation, 


$$
S^{*}=\bigcap\{S+m Z \mid m \in Z \backslash\{0\}\} .
$$

The closed sets of integers are exactly those sets $S$ for which $L(S)$ is residually finite!

THEOREM 1. The residually finite image $(L(S))_{f}$ of $L(S)$ is canonically isomorphic to $L\left(S^{*}\right)$, where $S^{*}$ denotes the closure of $S$ under the ideal topology of $S$. In particular $L(S)$ is residually finite if and only if $S$ is closed.

Proof. We show that $M_{f}(L(S))$ is the normal closure in $L(S)$ of the set $\left\{b_{i} b_{i}^{\prime} \mid i \in S^{*}\right\}$. First let $i \in S^{*}$, and let $\phi: L(S) \rightarrow G$ be any epimorphism onto a finite group. Then $a^{m}, a^{\prime m} \in \operatorname{Ker} \phi$ for some positive integer $m$. Now let $\alpha: Z \rightarrow Z / m Z$ be the canonical epimorphism. Then, by the definition of $S^{*}$, $s-i \in \operatorname{Ker} \alpha$ for some $s \in S$. Hence $b_{s} b_{i}, b_{s}^{\prime} b_{i}^{\prime} \in \operatorname{Ker} \phi$ and, since $b_{s} b_{s}^{\prime} \equiv 1$ in $L(S)$, we have $b_{i} b_{i}^{\prime} \in \operatorname{Ker} \phi$. Thus $b_{i} b_{i}^{\prime} \in M_{f}(L(S))$.

For the converse it suffices to show that $L(S)$ is residually finite whenever $S$ is closed. We apply Lemma 2 . with $G=L, U=J(S), \sigma=\sigma(S), N=\{N \triangleleft L \mid L / N$ finite $\}, H=L^{\prime}$, etc. Conditions (i),(ii) and (iii) are obviously satisfied and the crucial point is to verify (iv). Now either $S=Z$ or $Z \backslash S$ is infinite. But $J(Z)$ $=B \triangleleft L$ and $L / B \simeq A$ is residually finite, so (iv) holds if $S=Z$ and we may assume that $Z \backslash S$ is infinite. Then there are functions, $r, q: Z \rightarrow Z$ defined by

$$
\begin{gathered}
r(h)=\left\{\begin{array}{l}
1 \text { if } h \in S, \\
\text { the least } k \in Z \text { such that } k>|h| \text { and } h+k Z \subset Z \backslash S, \quad \text { if } h \notin S,
\end{array}\right. \\
\qquad q(m)=\prod_{h=-m}^{m} r(h) .
\end{gathered}
$$

Observe that the range of $q$ is an infinite set of positive integers. Set $N_{m}$ $=\left\langle\left\langle a^{q(m)}\right\rangle\right\rangle_{L}$. Then $N_{m} \in \mathscr{N}$ and to verify (iv) we show that $\bigcap_{m \in \omega} J(S) N_{m}$ $=J(S)$. Let

$$
w=a^{t} \prod_{i=-n}^{n} b_{i}{ }^{v i}
$$

choose $m \geqq n$ large enough so that $|t|<q(m)$ and $2 n<q(m)$ and assume that $w \in J(S) N_{m}$. Since $L / B \simeq A, J(S)<B$ and $|t|<q(m)$ we have $t=0$ and

$$
w v=\prod_{i=-n}^{n} b_{i}^{v_{i}} v \in J(S) \text { for some } v \in N_{m} \cap B .
$$

Now, by (12), $v$ written in reduced form on the $b_{j}$ 's is a product of words of the form $b_{j} b_{j+z q(m)}$. Thus, if $v_{i} \neq 1$, then either $b_{i}$ occurs in the reduced form of $w v$ or else it is replaced by $b_{i+z q(m)}$, for some $0 \neq z \in Z$, since by choice of $m$, $|i+z q(m)|>n$. But $i+z q(m) \in i+r(i) Z$, since $m \geqq|i|$, and so $i+z q(m) \notin S$ 
if $i \notin S$. Therefore, if $v_{i} \neq 1$ then $i \in S$ and so $w \in J(S)$. It follows that $w \in J(S) N_{m}$ implies $w \in J(S)$. Hence $\bigcap_{m \in \omega} J(S) N_{m}=J(S)$, all the conditions of Lemma 2 are satisfied, and $L(S)$ is residually finite.

Incidentally; this proof shows that the residually torsion groups $L(S)$ are residually finite.

The re structure of $L(S)$ is properly correlated with that of $S$, namely:

THEOREM 2. The group $L(S)$ is re, co-re or recursive if and only if $S$ has the corresponding property.

Proof.Because of the normal forms for $L$ and for amalgamated products, $i \in S$ if and only if $b_{i} b_{i}^{\prime} \equiv 1$ in $L(S)$, for all $i \in Z$.

Hence it is clear that $L(S)$ is re, co-re or recursive whenever $S$ is re, co-re or recursive, respectively. Conversely, if $S$ is re, then so is the entire set of defining relators for $L$ in terms of the generators, $a, a^{\prime}, b, b^{\prime}$ and so their normal closure in $F\left(a, a^{\prime}, b, b^{\prime}\right)$ is also re.

Now let $S$ be co-re. Then a recursive enumeration of $Z \backslash S$ gives rise to an enumeration of the set $T$ of all words of the form

$$
w=\alpha_{1} \beta_{1} \alpha_{1}^{\prime} \beta_{1}^{\prime} \cdots \alpha_{n} \beta_{n} \alpha_{n}^{\prime} \beta,
$$

where $\alpha_{i} \in A, \alpha_{i}^{\prime} \in A^{\prime}, \beta_{i} \in J(Z \backslash S), \beta_{j}^{\prime} \in J^{\prime}(Z \backslash S)$ and $\beta \in J^{\prime}(Z)=B^{\prime}$. Since (*) is just the normal form for the amalgamated product $L(S)$, every element of $L(S)$ is equal to exactly one word of $T$. In particular, 1 equals the empty word $\Lambda$. Thus we have the domain of $L(S)$, and hence also $L(S)-\{1\}$, represented by an re set. Note, however, that the multiplication of $L(S)$ is not represented by a recursive operation, e.g., if $0 \notin S$ then $w_{1}=a^{-k} b_{0} \in T$ and $w_{2}=a^{k} b_{k}^{\prime} \in T$, but $w_{1} w_{2} \equiv \Lambda$ or $b_{k} b_{k}^{\prime} \in T$ according as $k \in S$ or $k \notin S$. It remains to show that the set of all words on $\left\{a, a^{\prime}, b, b^{\prime}\right\}$ that do not belong to the normal closure of $\left\{b_{i} b_{i}^{\prime} \mid i \in S\right\}$ is re. This follows now from the fact that $L(S)$ is effectively residually recursive. More precisely, for any $w \neq \Lambda$ as in (*) set

$$
I_{w}=\left\{k \in Z \mid b_{k} \text { occurs in some } \beta_{i} \text { or } b_{k}^{\prime} \text { occurs in some } \beta_{j}^{\prime}\right\},
$$

and $S_{w}=Z \backslash I_{w}$. Then $S_{w}$ is co-finite and hence recursive. Thus the kernel $K_{w}$ $=\left\{u \in F\left\{a, a^{\prime}, b, b^{\prime}\right\} \mid u \equiv 1\right.$ in $\left.L\left(S_{w}\right)\right\}$ is re. But $w$ is clearly also in normal form for $L\left(S_{w}\right)$. Hence, for all $u \in K_{w}, w u \neq 1$ in $L\left(S_{w}\right)$ and therefore $w u \neq 1$ in $L(S)$, since $S \subset S_{w}$. It follows that $\bigcup\left\{w K_{w} \mid w \in T-\{\Lambda\}\right\}$ coincides with the set of all nontrivial words of $L(S)$. Since $T$ is re and the sets $K_{w}$ are re sets uniformly dependent on the $w^{\prime}$, this set is re.

Finally, if $S$ is recursive then by above $L(S)$ is both re and co-re hence recursive. So the theorem is proved. 


\section{The examples}

We denote by $P=\left\{p_{n} \mid n \in \lambda\right\}$ the sequence of rational primes in their natural order starting with $p_{0}=2$. Every finite set $S \subset Z$ is clearly closed and so is $Z$ itself.

$$
\text { If } S \subset P \text { then } S^{\prime}=S \cup\{-1,1\} \text { is closed. }
$$

In fact, the complement of of $S^{\prime}$ is the union of all sets of the form $m Z$ with $m \in P \backslash S$ or $-p+p^{2} Z$ with $p \in S$. Whether or not the closure of $S \subset P$ contains 1 or -1 depends on whether or not $S$ contains for every positive integer $m$ a prime congruent modulo $m$ to 1 or -1 , but this question does not concern us here. Let $R$ be any non-recursive re set, and let $S_{1}=\left\{p_{n} \in P \mid n \in R\right\}, S_{2}$ $=\left\{p_{n} \in P \mid n \notin R\right\}$. By Theorems 1. and 2. we have

(2) The groups $L\left(S_{1}^{\prime}\right)$ and $L\left(S_{2}^{\prime}\right)$ are both residually finite groups with unsolvable words problems. $L\left(S_{1}^{\prime}\right)$ is re and $L\left(S_{2}^{\prime}\right)$ is co-re.

It follows from Proposition 2. that these groups are neither finitarily embeddable into a finitely presentable group nor embeddable into a residually finite group that is finitely presentable as a residually finite group.

Now let $f: \omega \rightarrow \omega$ be a fixed recursive function whose range is not recursive and define the recursive function $g: \omega \times \omega \rightarrow \omega$ by

and

$$
g(n, 0)=0, \quad g(n, m+1)=g(n, m)+\|n-f(g(n, m))\|
$$

$$
g(n,-m)=-g(n, m) \text { for } m \in \omega, \text { where }\|0\|=0 \text { and }\|x\|=1 \text { for } x \neq 1 .
$$

(3) $g(n, m)=m$ if $n \notin \operatorname{Im} f$, the range of $f$,

$|g(f(k), m)| \leqq k$, for all $k \in \omega, m \in Z$.

We define $h: \omega \times \omega \rightarrow \omega$ by

$$
h(n, m)=m(1-\| n-f(g(n, m) \|) .
$$

(4) $h(n, m)=0$ if $n \notin \operatorname{Im} f$,

$h(f(k), m)=m$, for $|m| \geqq k$ and all $k \in \omega$.

The sets of integers $C$ and $D$ are now defined as follows:

$$
\begin{aligned}
& C=Z \backslash\left\{p_{n}+g(n, m) p_{n} ! \mid n \in \omega, m \in Z\right\} \\
& D=Z \backslash\left\{p_{n}+h(n, m) p_{n} ! \mid n \in \omega, m \in Z\right\} .
\end{aligned}
$$

Clearly $1,-1 \in C \cup D, 0 \notin C$ if and only if $f(0)=0$ and $0 \notin D$ if and only if $f(0) \neq 0$. For an arbitrary integer $t$ with $|t|>1$ we have the following situation: $t \notin C$ if and only if for one of its prime divisors, say $p_{k}$, an integer $r$ can be found 
such that $|r|<|t|,|r|=g(k,|r|)$ and $t-p_{k}=r p_{k} !$; similarly for $t \notin D$ where the function $h$ replaces $g$. It follows that

(5) $C$ and $D$ are recursive sets.

We claim that the closures of $C$ and $D$ are the following sets:

(6) $C^{*}=Z \backslash\left\{p_{n}+p_{n} ! Z \mid n \notin \operatorname{Im} f\right\}=\left\{p_{f(k)}+m p_{f(k)} ! \mid k \in \omega, f(j) \neq f(k)\right.$ for $\left.j<|m|\right\} \cup C$.

(7) $D^{*}=Z \backslash\left\{p_{f(k)}+m p_{f(k)} ! Z \mid k \in \omega, m \geqq k\right\}=\left\{p_{n} \mid n \notin \operatorname{Im} f\right\} \cup D$.

That these sets are closed is clear and that they include $C$ and $D$ respectively follows from (3) and (4). To prove (6) it remains to show that

$$
r=p_{f(k)}+g(f(k), m) p_{f(k)} ! \in C^{*} \text { for all } k \in \omega, m \in Z \text {. }
$$

Now let $q$ be an arbitrary positive integer and assume that

$$
r+q(2 k+1) p_{f(k)} !=p_{n}+g(n, j) p_{n} !
$$

for some $n \in \omega, j \in Z$. If $n=f(k)$ then $g(f(k), j)=g(f(k), m)+q(2 k+1)$. But by (3) $|g(f(k), j)| \leqq k$, for all $j \in Z$, and we have a contradiction. On the other hand, if $f(k)<n$, then the left-hand side of our equation is divisible by $p_{f(k)}$ but not the right side. Similarly we argue if $n<f(k)$. Thus we have found an integer in the residue class $r+q Z$ that does not belong to the complement of $C$. Therefore $Z \backslash C$ includes no open neighbourhood of $r$ and $r \in C^{*}$. To establish (7) we first observe that if $n \in \operatorname{Im} f$ and $k$ is the least natural number $x$ such that $n=f(x)$, then

$$
p_{n}+h(n, m) p_{n} != \begin{cases}p_{f(k)}, & \text { if }|m|<k \\ p_{f(k)}+m p_{f(k)} !, & \text { if }|m| \geqq k\end{cases}
$$

Thus by (4) it remains to show that $p_{n} \in D^{*}$ for $n \notin \operatorname{Im} f$. Assume that then $n \notin \operatorname{Im} f$ and that, for some $q \geqq 1$,

$$
p_{n}+q p_{n} !=p_{l}+h(l, m) p_{l} ! \text { for some } l \in \omega, m \in Z \text {. }
$$

By (4), $l \neq n$. But, if $n<l$, then the left side of the equation is divisible by $p_{n}$ and the right side is not. Similarly it follows that $l \nless n$. Therefore no residue class of the form $p_{n}+q Z$ is entirely contained in the complement of $D$ and we have shown that $p_{n} \in D^{*}$, which proves (7).

Clearly $C^{*}$ is re but not co-re and $D^{*}$ is co-re but not re. Now let $f$ be chosen so that its range is recursively inseparable from the range of a recursive function $s$ and set

$$
E=D \cup\left\{p_{n} \mid n \in \operatorname{Im} s\right\} .
$$

(7) implies that $E \subset D^{*}$. Hence $E^{*}=D^{*}$ and $\left\{p_{n} \mid n \in \operatorname{Im} f\right\} \subset Z \backslash E^{*}$, and we obtain 
(8) $E$ and $Z \backslash E^{*}$ are recursively inseparable re sets.

From Theorems 1, 2 and Section 1 now follows:

THEOREM 3. (i) $L(C)$ is finitely generated and has a solvable word problem. $L(C)_{f}$ is residually finite and re but has an unsolvable word problem. $L(C)$ is not finitarily embeddable in a finitely presentable group.

(ii) $L(D)$ is finitely generated and has a solvable word problem. $L(D)_{f}$ is residually finite and co-re but has an unsolvable word problem. Moreover $L(D)$ has an epimorphic image $L(E)$, with $L(E)_{f}=L(D)_{f}$, such that the set of trivial words of $L(E)$ is recursively inseparable from the set of non-trivial words of $L(E)_{f}$.

(iii) If $L(D)_{f}$ is embeddable in a group that is finitely presentable as a residually finite group, then the open sentence problem for the theory of finite groups is undecidable. If $L(E)$ is finitarily embeddable in a finitely presentable group, then the set of conditional equations true in all groups is recursively inseparable from the set of conditional equations that fail in some finite groups.

We conclude by observing that replacement of the ideal topology by the 2-adic topology leads to similar results for residually finite 2-groups.

\section{References}

[1] W. Boone, 'Certain simple unsolvable problems of group theory', Nedere. Akad. Wetensch. Math. 16 (1954), 213-237, 492-497 and 17 (1955), 252-256, 571-577.

[2] A. Cobham. 'Undecidability in group theory', Amer. Math. Soc. Notices 9 (1961), 406.

[3] Hall, P. 'Finiteness conditions for soluble groups', Proc. Roy. Soc. (3) 4 (1954), 419-436.

[4] G. Higman, 'Subgroups of finitely presented groups', Proc. Roy. Soc. A 262 (1961), 455-475.

[5] V. Huber-Dyson, 'The word problem and residually finite groups', Amer. Math. Soc. Notices 11 (1964), 743.

[6] A. I. Mal'cev, 'The undecidability of the elementary theory of finite groups', Dok Ak Nauk (1961), 1009-1012.

[7] A. Tarski, A. Mostowski and R. Robinson, Undecidable Theories, (North-Holland Publishing Co., Amsterdam, 1963.)

University of Illinois

Chicago Circle

Chicago, Illinois, 60680, U.S.A. 